\title{
ENERGY LEVEL POSITION OF Ni AND BAND OFFSETS IN $\mathrm{Zn}_{1-x} \mathrm{Cd}_{x} \mathrm{Se}: \mathrm{Ni}$ AND $\mathrm{ZnS}_{x} \mathrm{Se}_{1-x}: \mathrm{Ni}^{*}$
}

\author{
T. SURKOVA
}

Institute of Metal Physics RAS, Ural Department

Kovalevskaya 18, 620219 Ekaterinburg, GSP-170, Russia

W. Giriat

IVIC, Centro de Fisica, Apto 1827, Caracas 1010A, Venezuela

M. Godlewski, P. KaCzor, A.J. Zakrzewski

Institute of Physics, Polish Academy of Sciences

Al. Lotników 32/46, 02-668 Warszawa, Poland

\section{S. Permogorov and L. Tenishev}

A.F. Ioffe Physical-Technical Institute, RAS, 194021 St. Petersburg, Russia

Absorption and reflectivity measurements have been carried out for $\mathrm{Zn}_{1-x} \mathrm{Cd}_{x} \mathrm{Se}: \mathrm{Ni}$ and $\mathrm{ZnS}_{x} \mathrm{Se}_{1-x}$ :Ni solid solutions. Energy level positions of nickel $2+/ 1+$ charge state have been used for estimation of band offsets for the valence and conduction bands of $\mathrm{ZnCdSe} / \mathrm{ZnSe}$ and $\mathrm{ZnSSe} / \mathrm{ZnSe}$. Intra-shell transitions of $\mathrm{Ni}^{2+}$ were also studied.

PACS numbers: 71.55.Gs, 78.20.Wc

\section{Introduction}

It was pointed out $[1,2]$ that the energy levels of transition metal (TM) ions are pinned to a certain universal reference level of semiconductor. It was also shown that for two isovalent semiconductors the difference in the valence band-referred TM energy levels is equal to the valence band offsets of a given heterojunction, i.e., the band offsets can be estimated if the energy level positions of TM ions are known for given compounds. In this paper we positively verify the theory for $\mathrm{Ni}$ ion in solid solutions $\mathrm{Zn}_{1-x} \mathrm{Cd}_{x} \mathrm{Se}$ and $\mathrm{ZnS}_{x} \mathrm{Se}_{1-x}$ for the wide range of $\mathrm{Cd}$ and $\mathrm{S}$ fractions. The values of the band offsets which were extrapolated from the optical absorption data are very close to the theoretical ones predicted by the above-mentioned theory. We point out, however, that a relatively good agreement is also obtained from a simple model which takes into account differences in electron affinities.

*This work is partly supported by the Intern. Science Foundation Grant No. RG 0000. 


\section{Experimental}

The single crystals of $\mathrm{Zn}_{1-x} \mathrm{Cd}_{x} \mathrm{Se}: \mathrm{Ni}$ and $\mathrm{ZnS}_{x} \mathrm{Se}_{1-x}$ :Ni with Cd or S fractions from $x=0.0$ to 0.5 were grown by the chemical transport method. All samples were doped with nickel to the level of about $10^{17} \mathrm{~cm}^{-3}$. Absorption and reflection spectra were recorded by a BOMEM DA.13 Fourier transform spectrometer and DFS-12 monochromator. The samples were mounted in a gas flow liquid helium cryostat working in the temperature range of 2-300 K. Exciton reflectivity measurements were done to establish band gap energies, in which the position of maximum of a line was taken as a free exciton energy. The observation of broad, relatively strong absorption band in the range $1.7-2.2 \mathrm{eV}$ allowed us to estimate energy level positions of nickel $2+/ 1+$ charge state. These results are presented in Table.

TABLE

Composition $x$, position of a free exciton reflection maximum $E_{\mathrm{FE}}$ and a threshold energy $h \nu_{\text {th }}$ for the Ni photoionization $(2+\rightarrow 1+)$ in $\mathrm{Zn}_{1-x} \mathrm{Cd}_{x} \mathrm{Se}: \mathrm{Ni}$ and $\mathrm{ZnS}_{x} \mathrm{Se}_{1-x}: \mathrm{Ni}$ alloys at $2 \mathrm{~K}$.

\begin{tabular}{c|c|c|c}
\hline \hline Compound & $x$ & $E_{\mathrm{FE}}[\mathrm{eV}]$ & $h \nu_{\text {th }}[\mathrm{eV}]$ \\
\hline $\mathrm{ZnSe}: \mathrm{Ni}$ & 0.0 & 2.80 & 1.85 \\
\hline & 0.001 & 2.80 & 1.85 \\
& 0.01 & 2.78 & 1.85 \\
$\mathrm{Zn}_{1-x} \mathrm{Cd}_{x} \mathrm{Se}: \mathrm{Ni}$ & 0.1 & 2.69 & 1.85 \\
& 0.2 & 2.52 & 1.85 \\
& 0.3 & 2.44 & 1.81 \\
& 0.5 & $2.26^{*}$ & 1.82 \\
\hline & 0.001 & 2.80 & 1.85 \\
& 0.01 & 2.81 & 1.85 \\
$\mathrm{ZnS}_{x} \mathrm{Se}_{1-x}: \mathrm{Ni}$ & 0.1 & 2.86 & 1.90 \\
& 0.2 & 2.93 & 1.95 \\
& 0.5 & $3.2^{*}$ & 2.07 \\
\hline
\end{tabular}

${ }^{*}$ These values are calculated from the $E_{\mathrm{FE}}(x)$ dependence.

\section{Discussion}

The threshold energies were determined from $\alpha^{2 / 3}$ versus $h \nu$ plot, which should be a straight line for the photoionization process [3], cutting the energy axis at the impurity ionization energy. The value $h \nu=1.85 \mathrm{eV}$ for ZnSe:Ni crystal is in agreement with some previous estimations [3]. For $\mathrm{ZnCdSe:Ni} \mathrm{this} \mathrm{energy}$ does not change to $x=0.2$, and then decreases by ca. $30 \mathrm{meV}$ for a larger Cd content. The energy level position of $\mathrm{Ni}$ in $\mathrm{ZnSSe}: \mathrm{Ni}$ increases monotonously by about $200 \mathrm{meV}$ for the range of $S$ fraction varied between 0.01 and 0.5 . Taking the $\mathrm{Ni}$ acceptor level as a reference level we estimated valence and conduction band edges in the whole composition range studied (Fig. 1). The values of the 
determined band offsets are very close to the theoretical ones [2]. A zero valence band offset and a large conduction band offset were predicted by the common anion rule for the $\mathrm{ZnCdSe} / \mathrm{ZnSe}$ heterojunction [4]. The weak shift of the valence band edge for $x>0.2$ (Cd) can be connected with a structural transition of a crystal from a zinc-blend to a wurzite phase, which takes place in this composition range. Our data for the $\mathrm{ZnSSe} / \mathrm{ZnSe}$ show almost equal valence and conduction band offsets. They are relatively close to those obtained from a simple electron affinity rule [5], but differ from a number of experimental data for $\mathrm{ZnSSe} / \mathrm{ZnSe}$ superlattices, which give for the conduction band offset values in the range of $0.01-0.0688 \mathrm{eV}$ [5]. We can suggest that in solid solutions with anion substitution the ionization energy of a TM impurity can depend on a composition, because in this case the nearest-neighbor impurity surrounding and, thus hybridization of the impurity $d$-shell and of the valence band $p$-states, changes strongly.

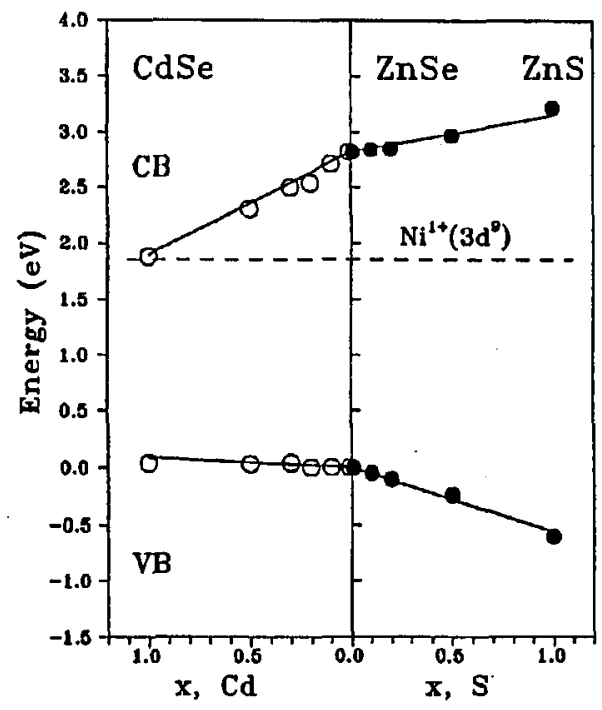

Fig. 1. The valence and conduction band offsets of $\mathrm{ZnCdSe} / \mathrm{ZnSe}$ and $\mathrm{ZnSSe} / \mathrm{ZnSe}$ measured relative to the $\mathrm{Ni}$ acceptor level. Solid line is extrapolated from the TM everaging procedure for the corresponding binary compounds (after Ref. [2]).

Intra-shell transitions of $\mathrm{Ni}^{2+}\left(3 d^{8}\right)$ are also modified with the change of $x$ composition. The disorder of the alloys produces qualitatively similar broadening of the absorption lines in compounds with substitution in the anion and cation sublattices, but in $\mathrm{ZnSSe}: \mathrm{Ni}$ this broadening is more pronounced. Absorption lines also shift with increasing $x$ composition. The magnitude and sign of the shifts are different for each transition. For example, for the ${ }^{3} T_{1}(F) \rightarrow{ }^{1} T_{2}(F)$ transition the line shifts slightly to the lower energy in ZnCdSe:Ni (by about $40 \mathrm{~cm}^{-1}$ for $x=0.1$ ) and to the higher energy in ZnSSe:Ni (by about $15 \mathrm{~cm}^{-1}$ for $x=0.1$ ). The temperature induced modification of the spectra was also studied. 
Concluding, we have determined energy level positions of $\mathrm{Ni} 2+/ 1+$ charge state for the wide range of $\mathrm{Cd}$ and $\mathrm{S}$ content in $\mathrm{ZnCdSe}$ and $\mathrm{ZnSSe}$. The estimated band offsets for the valence and conduction bands of $\mathrm{ZnCdSe} / \mathrm{ZnSe}$ and $\mathrm{ZnSSe} / \mathrm{ZnSe}$ are in good agreement with the theory [1, 2], but in the latter case they differ from some experimental data obtained for superlattices.

\section{References}

[1] J.M. Langer, H. Heinrich, Phys. Rev. Lett. 55, 1414 (1985).

[2] J.M. Langer, C. Delerue, M. Lannoo, H. Heinrich, Phys. Rev. B 38, 7723 (1988).

[3] J.M. Noras, J.W. Allen, J. Phys. C, Solid State Phys. 13, 3511 (1980).

[4] K.P. O'Donnell, P.J. Parbrook, B. Henderson, C. Trager-Cowan, X. Chen, F. Yang, M.P. Halsall, P.J. Wright, S. Cockayne, J. Cryst. Growth 101, 554 (1990).

[5] P.J. Parbrook, K.P. O'Donnell, in: II-VI Semiconductor Compounds, Ed. M. Jain, World Scientific, Singapore 1993, p. 255. 